\title{
Effets du glucose et de l'arginine sur la sécrétion d'insuline au cours des syndromes de Cushing
}

\author{
E. Modigliant, G. Strauch, J. P. Luton, H. Bricatre
}

Laboratoire de Pathologie Médicale de la Faculté de Médecine et Service d'Endocrinologie et de Métabolisme (Pr. H. Bricaire) - Hopital Cochin - Paris XIVe

\section{Reçu le 13 janvier 1969}

\begin{abstract}
Effects of Glucose and Arginine on the Secretion of Insulin in Cushing's Syndrome

Summary. Plasma insulin levels have been measured by radio-immunoassay in 15 patients with. untreated Cushing's syndrome, using oral glucose tolerance tests (O.G.T.T.), arginine infusions (A.T.) or both of these stimuli. During O.G.T.T., glucose tolerance was impaired in 12 subjects, at the upper limit of the normal range in two, and normal in only one case. Statistically significant hyperinsulinaemia occured in 5 patients. Nine subjects had plasma insulin levels in the control's range, though their glycaemic curve was of the diabetic type. During arginine infusion, insulin peak was not reduced in these 9 patients. A negative response to each test was observed in one patient. These heterogeneous results are discussed in the light of experimental data and known physiological effects of glucocorticoids.
\end{abstract}

Résumé. L'insulinémie a été étudiée avant tout traitement chez 15 malades atteints d'un syndrome de Cushing lors de la stimulation par le glucose oral, l'arginine intraveineuse ou les deux. Les courbes glycémiques étaient de type diabétique dans 12 cas, à la limite supérieure de la normale chez deux patients et normale dans un seul cas. Cinq de ces sujets seulement ont un hyperinsulinisme statistiquement significatif lors de l'hyperglycémie orale. Neuf ont une insulinémie dans les limites de la normale alors que leurs courbes glycémiques sont diabétiques. Le pic insulinémique lors de l'épreuve à l'arginine n'est pas diminué chez ces derniers. Un malade enfin a une réponse insulinémique très faible au cours des deux épreuves. L'hétérogénéité des troubles de l'insulino-secrétion observée au cours du syndrome de Cushing est discutée à la lumière des données expérimentales et des effets physiologiques connus des glucocorticoïdes.

Einfluß von Glucose und Arginin auf die Insulinselsretion beim Cushing-Syndrom

Zusammenfassung. Bei 15 Cushing-Patienten wurden die Seruminsulinspiegel während einer oralen Glucosebelastung und einer Arginin-Infusion untersucht. 12 der Patienten zeigten eine eingeschränkte, 2 eine leicht gestörte und nur 1 Patient eine normale Glucosetoleranz. 5 Patienten hatten wäbrend der oralen Glucosebelastung eine statistisch signifikante Hyperinsulinämie; bei 9 lag die Insulinkonzentration des Blutes innerhalb normaler Grenzen, obgleich sich eine pathologische Blutzuckerkurve fand. Die Maximalwerte der Seruminsulinspiegel unter Arginin-Infusion waren bei diesen 9 Patienten nicht verringert. Bei einem Patienten führten beide Belastungen nur zu einem minimalen Anstieg der Seruminsulinspiegel. Diese heterogenen Befunde zur Insulininkretion beim Cushing-Syndrom werden unter Berücksichtigung experimenteller Befunde und der bekannten physiologischen Effekte der C్tlucocorticoide besprochen.

Key-words: Cushing's syndrome, insulin (blood, secretion), hyperinsulinism, amino-a.cids, glucose tolerance test, radio-immuno assay, diabetes, obesity.
Les troubles de la glycorégulation sont habituels dans le syndrome de Cushing $[22,28,34,36]$. L'augmentation de la néoglycogénèse ainsi que celle du débit hépatique du glucose secondaires à l'hypersécrétion de cortisol font partie des explications proposées [7, 20].

Les publications faisant état de dosages d'insulinémie au cours $d u$ syndrome de Cushing sont rares [33, $15,16]$. Elles ont montré, lors de la charge orale en glucose, un hyperinsulinisme qui, pour certains, serait en rapport avec l'obésité [15].

Nous rapportons ici notre expérience des variations insulinémiques chez quinze sujets présentant un syndrome de Cushing, peu ou non obèses, étudiés à l'aide de deux stimuli secrétoires agissant par des mécanismes différents [11]: la charge glucosée orale et la perfusion d'arginine.

\section{Materiel et Méthodes}

A) Le groupe des patients comprend quatre hommes et onze femmes, dont les âges extrêmes vont de 23 à 57 ans et dont l'âge moyen est de 40 ans (Tableau 1). L'exploration a été faite avant traitement chez tous les sujets; avant et après chez deux d'entre eux. Les étiologies se répartissent de la façon suivante:

10 maladies de Cushing par hyperplasie surrénale bilatérale d'origine centrale

2 corticosurrénalomes malins

2 adénomes bénins

1 tumeur hypophysaire exteriorisée

Le diagnostic a été posé dans tous les cas sur des arguments biologiques (absence de freinage du cortisol plasmatique et des 17 hydroxy-corticoides par la déxaméthasone) et radiographiques (retro-pneumopéritoine ou artériographie). Un traitement chirurgical a été pratiqué dans 11 cas (ablation de l'adénome ou du carcinome, ou surrénalectomic totale bilatérale en cas d'hyperplasie) et a permis la confirmation histologique. Trois malades sont actuellement en cours de préparation à l'intervention par une chimiothérapie antisurrénalienne par l'OPDDD ${ }^{1}$ (IV, X, XIII). Une malade a été traitée par radiothérapie hypophysaire (VII).

1 OP'DDD: ortho para prime (dichlorodiphényldichloréthane). 
B) L'insulinémie chez ces malades a été étudiée

treize fois au cours d'une hyperglycémie orale $(1 \mathrm{~g}$ de glucose par kilo de poids), les prélévements étant faits généralement toutes les trente minutes ${ }^{2}$. L'épreuve fut prolongée jusqu'à la cinquième heure dans huit cas, jusqu'à la quatrième heure dans trois cas, jusqu'à la troisième heure dans deux autres cas. Deux malades (I, XIII) ont été à nouveau explorés après traitement.
Une hyperglycémie provoquée a été pratiquée chez 11 sujets normaux ( 2 hommes, 9 femmes) dont l'âge moyen était de 35 ans (les âges extrêmes se situant entre 16 et 59 ans).

Une perfusion d'arginine fut également pratiquée chez 17 sujets témoins, 4 hommes et 13 femmes, dont l'âge moyen était de 47 ans (les âges extrêmes se situaient entre 20 et 70 ans). Les moyennes des résul-

Tableau 1. Principaux caractères cliniques, histologiques et biologiques de 15 patients présentant un syndrome de Cushing

\begin{tabular}{|c|c|c|c|c|c|c|c|}
\hline No & Age & Poids & Taille & Cause & $\begin{array}{l}\text { H.G.P. } \\
\text { Diabétique }\end{array}$ & $\mathrm{I} / \mathrm{G}$ & $\begin{array}{l}\text { Delta sous } \\
\text { Arginine }\end{array}$ \\
\hline I & 41 & 65 & 158 & A.S. & + & 1.42 & \\
\hline II & 46 & 76 & 166 & H.B.S. & + & $\begin{array}{l}1 . \pm 2 \\
1.1\end{array}$ & 44 \\
\hline III & 55 & 67 & 166 & H.B.S. & + & 0.09 & 44 \\
\hline IV & 37 & 72 & 169 & H.M.A. & + & 0.54 & 52 \\
\hline $\mathrm{V}$ & 46 & 64 & 160 & H.B.S. & + & 1.15 & \\
\hline VI & 23 & 54 & 164 & H. M. A. & $\lim$ & 2.24 & 237 \\
\hline VII & 36 & 53 & 159 & H.B.S. & - & 3.40 & 86 \\
\hline VIII & 27 & 71 & 176 & H.B.S. & $\lim$ & 0.47 & \\
\hline $\mathrm{IX}$ & 24 & 65 & 162 & H.B.S. & + & 1.95 & 183 \\
\hline $\mathrm{X}$ & 48 & 75 & 169 & A.S. & + & 0.93 & 76 \\
\hline XI & 35 & 58 & 159 & H.M.A. & + & & 103 \\
\hline XII & 27 & 52 & 167 & K.S. & $i$ & 0.65 & \\
\hline XIII & 57 & 80 & 158 & H.B.S. & + & 0.02 & 15 \\
\hline XIV & 45 & 62 & 170 & K.S. & & & 40 \\
\hline$X V$ & 46 & 52 & 159 & A. H. & + & & 106 \\
\hline
\end{tabular}

$\begin{array}{ll}\text { I/G: } & \text { Rapport Insuline/Glucose } \\ \text { K.S. } & \text { Cancer Surrénalien } \\ \text { A.S.: } & \text { Adénome Surrénalien } \\ \text { H.B.S.: } & \text { Hyperplasie Bilatérale des Surrénales } \\ \text { H.M.A.: } & \text { Hyperplasie Micro-Adénomateuse } \\ \Delta \text { insulinémique: } & \text { différence entre la valeur maxima obtenue au cours de l'épreuve et l'insulinémie basale. } \\ \text { H.G.P. diabétique: } & \text { flêche supérieure à } 180 \mathrm{mg} / 100 \mathrm{ml} \text { et glycémie à } 120 \mathrm{~min} \text { supérieure à } 140 \mathrm{mg} . \\ \text { H.G.P. limite: } & \text { sous entend une seule de ces deux anomalies. }\end{array}$

Onze fois au cours d'une épreuve comportantla perfusion en $30 \mathrm{~min}$, à débit constant, d'une solution aqueuse de monochlorhydrate d'arginine. Les trois patients initialement explorés (III, IX, XIV) reçurent une dose fixe de $25 \mathrm{~g}$ (ce qui correspondait à une dose de $0,4 \mathrm{~g} / \mathrm{kg}$ ); les autres reçurent une dose de $0.5 \mathrm{~g} / \mathrm{kg}$ de poids. Afin de doser simultanément l'hormone de croissance plasmatique, deux des trois sujets masculins (IX, X) furent traités par $5 \mathrm{mg}$ de diéthylstilboestrol au cours des quarante huit heures précédant le test. Les prélévements pour insulinémie ont été effectués aux temps $0-5-15-30-60-90-120 \mathrm{~min}$.

Neuf des quinze patients subirent une double exploration par hyperglycémie provoquée et perfusion d'arginine.

Tous les tests ont été faits chez des sujets à jeun et au repos depuis douze heures, le sang veineux étant recueilli sur E.D.T.A. puis centrifugé et le plasma conservé à $-20^{\circ} \mathrm{C}$.

Les quinze malades explorés ont été comparés à un certain nombre d'épreuves faites dans les mêmes conditions chez des sujets témoins.

2 Certains prélévements n'ont pu être obtenus en raison des difficultés de ponction veineuse chez ces malades. tats obtenus chez les témoins sont indiquées aux tableaux 2 et 3 .

\section{C) Méthodes}

L'insulinémie a été dosée par la méthode radioimmunologique en utilisant les propriétés d'adsorption du traceur libre au talc selon la technique précédemment décrite $[23,32]$.

Les résultats sont exprimés en micro-unités par millilitres ${ }^{3}$.

Les glycémies ont été dosées à l'auto-analyseur par la méthode au ferri-cyanure.

Les moyennes sont présentées avec leurs écarttypes.

\section{Résultats}

I. L'insulinémie basale fut retrouvée dans les limites normales 23 fois sur 26 .

\section{Hyperglycémie Provoquée}

a) malades non traités

1. Glycémies

deux patients ont une glycémie basale élevée: 1.20 (IV) et $2.20 \mathrm{~g} / \mathrm{l}$ (XIII),

3 Le standard d'insuline humaine, titrant $24.3 \mathrm{UI} / \mathrm{mg}$, a été mis à notre disposition par les Laboratoires novo. 
Tableau 2. Réponses glycémiques $(B)$ et insulinémiques (A) au glucose oral chez 13 patients atteints de Syndrome de Cushing:

A. Insulinémies en $\mu \mathrm{U} / \mathrm{ml}$

\begin{tabular}{|c|c|c|c|c|c|c|c|c|c|}
\hline & 0 & 30 & 60 & 90 & 120 & 150 & 180 & 240 & 300 \\
\hline $\bar{I}$ & 12 & 182 & 125 & 91 & 105 & 107 & 47 & 20 & 20 \\
\hline II & 8 & 34 & 68 & & 116 & 32 & 24 & 16 & 8 \\
\hline III & 14 & 33 & 30 & 35 & 25 & 14 & 20 & 26 & 14 \\
\hline IV & 16 & 36 & 46 & & 102 & 124 & 60 & 24 & \\
\hline V & 39 & 150 & 150 & 110 & 150 & 75 & 56 & 26 & 34 \\
\hline VI & 21 & 90 & 105 & 132 & 150 & 67 & 87 & 32 & 18 \\
\hline VII & 10 & 56 & 300 & & 150 & 50 & 34 & 8 & 8 \\
\hline VIII & 25 & 31 & 64 & & 42 & 55 & 8 & & \\
\hline IX & 12 & 200 & 185 & 180 & & 170 & 120 & 20 & $\mathbf{3}$ \\
\hline $\mathrm{X}$ & 8 & 50 & 110 & 76 & 88 & 50 & 30 & 16 & 8 \\
\hline XI & 8 & & 44 & & & 28 & 20 & 8 & \\
\hline XII & 5 & 28 & 55 & & 39 & & 32 & & \\
\hline XIII ${ }^{a}$ & 8 & 22 & 8 & & 8 & & 8 & 8 & \\
\hline Moy. & $16 \pm 9$ & $88 \pm 62$ & $111 \pm 75$ & 104 & $93 \pm 50$ & $72 \pm 46$ & $50 \pm 26$ & $18 \pm 8$ & $15 \pm 9$ \\
\hline $\begin{array}{l}\overline{\text { Moy. } 11} \\
\text { Témoins }\end{array}$ & $10 \pm 4.8$ & $67 \pm 35$ & $52 \pm 37$ & & $40 \pm 30$ & $19 \pm 19$ & $11 \pm 6.1$ & $9 \pm 9$ & - \\
\hline $\begin{array}{l}\text { Apres Tt. } \\
\text { XIII } \\
\text { I }\end{array}$ & $\begin{array}{l}8 \\
7\end{array}$ & $\begin{array}{r}8 \\
66\end{array}$ & $\begin{array}{l}20 \\
38\end{array}$ & 24 & $\begin{array}{r}8 \\
22\end{array}$ & $\begin{array}{r}8 \\
24\end{array}$ & 8 & 8 & \\
\hline
\end{tabular}

a les résultats du sujet $n^{\circ}$ XIII ne sont pas inclus dans la moyenne.

B. $\Delta$ Glycémiques en $\mathrm{mg} / 100 \mathrm{ml}$

\begin{tabular}{|c|c|c|c|c|c|c|c|c|c|}
\hline & 0 & 30 & 60 & 90 & 120 & 150 & 180 & 240 & 300 \\
\hline I & 68 & 42 & 76 & 83 & 104 & 76 & 40 & 16 & 10 \\
\hline II & 94 & 72 & 87 & - & 54 & 18 & -10 & -34 & -38 \\
\hline III & 74 & 72 & 158 & 186 & 160 & 118 & 92 & 52 & 20 \\
\hline IV & 20 & 85 & 130 & - & 135 & 95 & 20 & -60 & -55 \\
\hline V & 56 & 84 & 86 & 80 & 76 & 52 & 18 & 6 & 0 \\
\hline VI & 80 & 45 & 30 & 35 & 40 & 25 & 10 & -5 & -5 \\
\hline VII & 50 & 58 & 115 & & 20 & -22 & -19 & -24 & -14 \\
\hline VIII & 73 & 57 & 77 & & 35 & 15 & 9 & & \\
\hline $\mathrm{IX}$ & 100 & 88 & 120 & 118 & 92 & 38 & -10 & -55 & -30 \\
\hline $\mathrm{X}$ & 64 & 60 & 86 & 88 & 87 & 40 & 0 & -16 & -4 \\
\hline XI & 60 & 46 & 92 & & 85 & 58 & 20 & -10 & 0 \\
\hline XII & 98 & 56 & 96 & & 46 & & -10 & & \\
\hline XIII & 252 & 16 & 114 & 168 & 188 & 198 & 136 & 933 & 38 \\
\hline Moy. & & $64 \pm 15.4$ & $96 \pm 30.7$ & $98 \pm 48.4$ & $80 \pm 39$ & $43 \pm 38$ & $18 \pm 28$ & $-17 \pm 30$ & $-11 \pm 21$ \\
\hline $\begin{array}{l}\text { Moy. } 11 \\
\text { témoins }\end{array}$ & & $34.5 \pm 16$ & $17 \pm 27$ & - & $8 \pm 14$ & $-1 \pm 11$ & $-5 \pm 14$ & $-10 \pm 16$ & \\
\hline \multicolumn{10}{|l|}{ Après Tt } \\
\hline I & & $\begin{array}{l}31 \\
85\end{array}$ & & 20 & 25 & $\begin{array}{r}25 \\
170\end{array}$ & & & \\
\hline XIII & & 85 & 125 & - & 145 & 170 & 125 & 85 & 85 \\
\hline
\end{tabular}

Tableau 3. Variations glycémiques et insulinémiques au cours de l'épreuve à l'arginine

Insulinémios $(\mu \mathrm{U} / \mathrm{ml}$

Deltas glycémiques $(\mathrm{mg} / 100 \mathrm{ml})$

\begin{tabular}{|c|c|c|c|c|c|c|c|c|c|c|c|c|c|}
\hline & 0 & 5 & 15 & 30 & 60 & 90 & 120 & $\mathbf{5}$ & 15 & 30 & 60 & 90 & 120 \\
\hline II & 8 & 28 & 44 & 42 & 52 & 8 & 8 & 6 & 17 & 20 & 27 & 10 & -4 \\
\hline III & 36 & & 52 & 80 & & 29 & & - & - & 26 & 0 & -2 & -20 \\
\hline IV & 8 & 60 & & 40 & 26 & & 18 & 5 & 20 & 25 & 20 & 5 & 5 \\
\hline VI & 13 & & 250 & 250 & 110 & 40 & 41 & - & 40 & 35 & 5 & 0 & 5 \\
\hline VII & 8 & 56 & 80 & 94 & 54 & 10 & 8 & 7 & 14 & 20 & 14 & -4 & -14 \\
\hline IX & 17 & 200 & 125 & 135 & 110 & 60 & 32 & 26 & 10 & 48 & 14 & 36 & 8 \\
\hline $\mathrm{X}$ & 8 & 40 & & 84 & 26 & 8 & 8 & 3 & 6 & 11 & 0 & -2 & -3 \\
\hline XI & 7 & 20 & 110 & 50 & 20 & & 25 & 12 & 14 & 18 & 3 & 2 & \\
\hline XIII ${ }^{a}$ & 8 & 24 & 8 & 8 & 8 & 8 & 8 & 26 & 46 & 54 & 64 & 54 & 34 \\
\hline XIV & 23 & & 23 & 40 & 38 & 20 & 16 & & 18 & 36 & 24 & 6 & 10 \\
\hline $\mathrm{XV}$ & 20 & 126 & 120 & & 72 & 60 & & 10 & 11 & 30 & 24 & 10 & 6 \\
\hline Moy. & $17 \pm 10$ & $70 \pm 58$ & $100 \pm 66$ & $91 \pm 63$ & $51 \pm 33$ & $32 \pm 19$ & $19 \pm 13$ & $10 \pm 7$ & $16 \pm 11$ & $26 \pm 10$ & $13 \pm 9.8$ & 86 土 11 & $-2 \pm 10$ \\
\hline $\begin{array}{l}\text { Ioy. } 17 \\
\text { Cem. }\end{array}$ & $9 \pm 5$ & $53 \pm 55$ & $61 \pm 43$ & $60 \pm 26$ & $36 \pm 24$ & $15 \pm 9$ & $9 \pm 4$ & $7 \pm 6$ & $14 \pm 11$ & $14 \pm 16.6$ & $9+17$ & $7-3 \pm 8.9$ & $0.6 \pm 6$ \\
\hline
\end{tabular}

a Les résultats du patient XIIT ne sont pas inclus dans la moyenne. 
dix ont une hyperglycémie anormale avec flèche élevée et retour à la normale retardé,

deux ont des chiffres limites à la deuxième heure (entre 1.20 et $1.40 \mathrm{~g} \%$ ) (VI, VIII),

une seule a une hyperglycémie provoquée normale (VII), selon les critères de Conn [30],

dans les onze cas où la glycémie a été dosée à la quatrième heure, une chute glycémique au moins égale à $20 \%$ de la valeur de base a été observée chez cinq sujets (II, IV, VII, IX, X).

\section{Insulinémies}

La comparaison des moyennes insulinémiques entre malades et témoins permet les constatations suivantes (Fig. 1, Tableau 2):

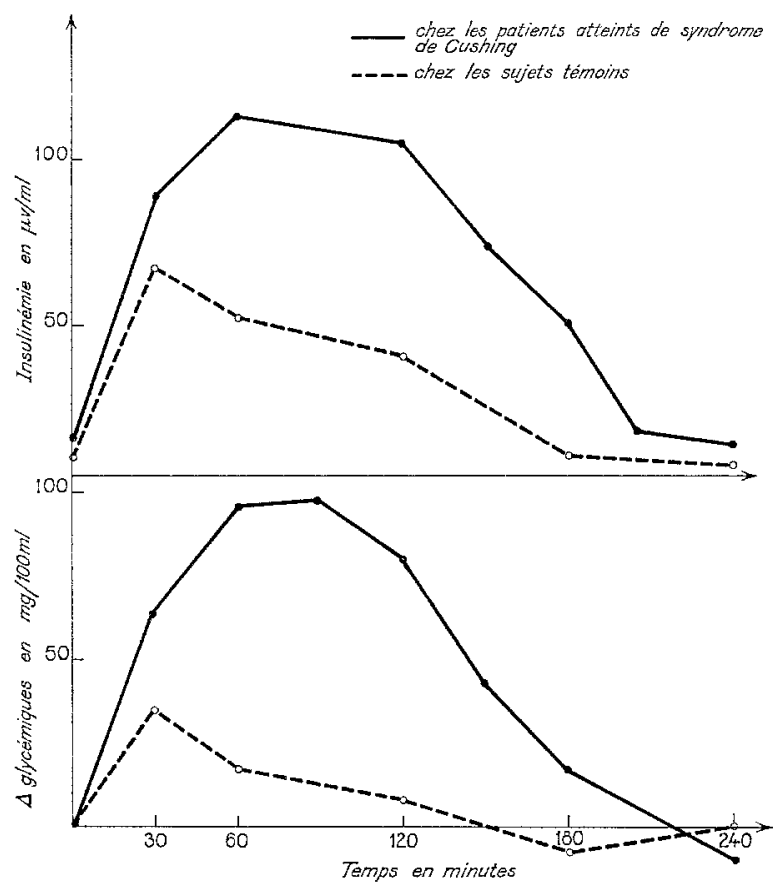

Fig. 1. Insulinémies et glycémies au cours de l'Hyper. glycémie orale

- chez les patients atteints de Syndrome de Cushing $\cdot-\cdots \cdot$ chez les sujets témoins

le delta insulinémique ${ }^{4}$ est retardé et sa valeur moyenne plus élevée que celle des témoins $(90 \mu \mathrm{U} \pm 32$ au lieu de $65 \pm 30$ ).

Les moyennes insulinémiques sont plus élevées à chaque temps sans que l'on puisse noter de différence significative avec les témoins.

L'insulinémie revient plus lentement à la normale que chez les témoins.

L'augmentation insulinique moyenne ${ }^{5}$ est de $320 \mu \mathrm{U}$ au lieu de $108 \mu \mathrm{U}$ chez les sujets normaux.

4 Delta insulinémique: différence entre la valeur maxima obtenue au cours de l'épreuve et l'insulinémie basale.

5 Augmentation insulinique: somme des augmentations par rapport à l'insulinémie basale aux temps $0-30-$ $60-90-120-180$.
L'analyse des différentes épreuves montre que les réponses obtenues sont hétérogènes; trois groupes se dégagent en effet:

cinq malades sur treize ont un pic significativement plus élevé $(P<0.05)$ que celui des témoins $(\mathrm{I}, \mathrm{V}, \mathrm{VI}$, VII, IX). Quatre d'entre eux (I, V, VI, IX) ont également une insulinémie à $150 \mathrm{~min}$ significativement supérieure aux témoins $(P<0.05)$. L'hyperglycémie est perturbée dans trois cas, limite dans un cas, normale dans le dernier. Il est à noter, en outre, que dans ce groupe, deux patients ont un pic insulinémique qui précède le pic glycémique ce que nous n'avons pas constaté chez les témoins (pic insulinémique à $30 \mathrm{~min}$, pic glycémique à 60 et $120 \mathrm{~min}$ chez les patients I et IX respectivement).

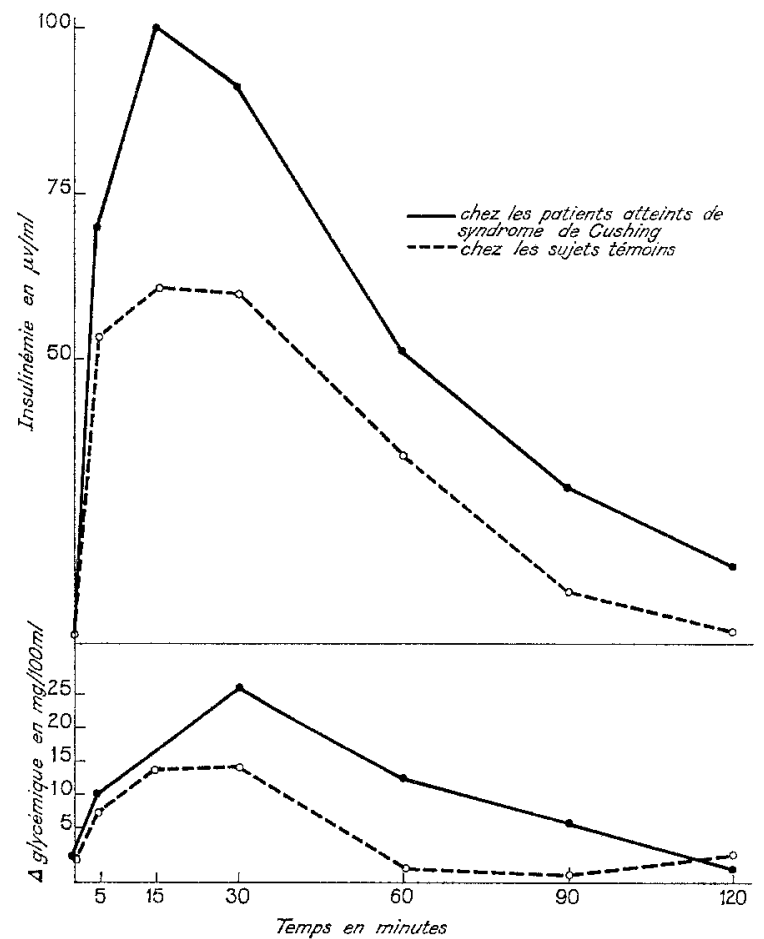

Fig. 2. Réponses insulinémiques et glycémiques lors du test à l'arginine

- - chez les patients atteints de syndrome de Cushing - - - chez les sujets témoins

Une seule malade (XIII) a une réponse hormonale très faible allant de pair avec un diabète franc.

Les autres ont un delta insulinémique dans les limites de la normale. L'hyperglycémie est de type diabétique dans six cas, limite chez le septième malade (VII). Quatre de ces patients ont un rapport insuline/ glucose $^{6}$ inférieur àcelui des témoins $(0.65$, alors que la limite inférieure des témoins est de 0.95).

Deux malades ont été explorés à nouveau après traitement: l'une $(\mathbf{I})$, opérée d'un adénome bénin, a

6 Rapport insuline/glucose: surface d'augmentation insulinique rapportée à la surface d'augmentation glycémique pendant les trois premières heures. 
actuellement une hyperglycémie normale la réponse insulinique s'est également normalisée - l'autre (XIII) traitée par l'OP'DDD ${ }^{7}$ a actuellement une glycémie basale à $1 \mathrm{~g}^{0} / 00$ au lieu de 2.20 mais l'hyperglycémie reste perturbée et la réponse insulinique est inchangée.

III. Perfusion d'arginine (Tableau 3, Fig. 2)

Si l'on excepte la malade diabétique (XIII) dont la réponse insulinique est très faible, l'insulinémie est dans l'ensemble plus élevée que chez les témoins. Le delta, qui se situe à quinze minutes comme chez les sujets normaux, est de $95 \mu \mathrm{U} \pm 64$ (moyenne chez 18 témoins $60 \mu \mathrm{U} \pm \mathbf{4 5}$ ).

Seuls deux malades sur onze ont un delta significativement plus élevé $(P<0.05)$. Le retour à la normale est légèrement retardé.

Quant à la glycémie, elle subit une élévation plus nette que chez les témoins $=0.25 \mathrm{~g} / 1$ au lieu de 0.16 . Le retour à la normale est retardé et la courbe glycémique n'a pas l'évolution diphasique habituelle chez les témoins.

La réponse insulinique à l'arginine est souvent parallèle au rapport insuline/glucose sous hyperglycémie provoquée (Tableau 1).

\section{Discussion}

Le Diabète ou du moins les perturbations de l'hyperglycémie provoquée, sont très fréquentes dans le syndrome de Cushing.

a) En effet, dans l'expérience des auteurs [4] portant sur 83 cas (Tableau 4), $40 \%$ ont une glycémie à jeun supérieure à $1.10 \mathrm{~g} \%, 24 \%$ ont une glycosurie et $71 \%$ une hyperglycémie provoquée de type diabétique (flèche $>1.80$ dans $76 \%$ des cas, glycémie à $120 \mathrm{~min}>$ $1.40 \mathrm{~g} \%$ dans $71 \%$ ).

Tableau 4. Fréquence des troubles de la glycorégulation dans le Syndrome de Cushing

\begin{tabular}{|c|c|c|c|c|}
\hline & NB & $\begin{array}{l}\text { Glycémie } \\
\text { à jeun } \\
\text { Elevée \% }\end{array}$ & $\begin{array}{l}\text { Glycosurie } \\
\%\end{array}$ & $\begin{array}{l}\text { Perturbations } \\
\text { de } I^{\prime} H . G . P \text {. } \\
\%\end{array}$ \\
\hline Thorn & 36 & 33 & 38 & 81 \\
\hline Sprague & 67 & 33 & & 52 \\
\hline Plotz & 33 & 15 & & 79 \\
\hline Soffer & 40 & 28.5 & & 52.5 \\
\hline $\begin{array}{l}\text { Mac } \\
\text { Cullagh }\end{array}$ & & & & \\
\hline $\begin{array}{l}\text { Cullagh } \\
\text { Bricaire }\end{array}$ & 34 & 20.6 & & 41.2 \\
\hline et coll. & 83 & 39.6 & 24.4 & 71 \\
\hline Total & 293 & $34 \%$ & $31.2 \%$ & $62.8 \%$ \\
\hline
\end{tabular}

b) La fréquence des troubles du métabolisme glucidique dans les autres statistiques de la littérature est similaire $[22,28,29,35,37]$.

c) D'après Miller [22], le diabète s'améliore presque toujours et disparaît dans plus de $50 \%$ des cas où le traitement a été conduit avec succès, le retour à la normale de l'hyperglycémie étant souvent plus long.

7 OP'DDD: orthopara prime (dichlorodiphényl-dichloréthane), chimiothérapie anti surrénalienne. d) A l'opposé, le diabète stéroidien iatrogène est rare puisqu' il survient dans moins de $1 \%$ des cas (Miller [22], Lebon [19]) et pratiquement toujours chez des malades ayant des antécédents personnels ou familiaux de diabète. Ceci s'explique peut-être par les données expérimentales: l'administration de Cortisone à des hamsters entraîne une élévation glycémique au bout de quelques jours, mais celle-ci tend à disparaître sauf si l'on augmente les doses par paliers [5]. ing

L'insulino-sécrétion au cours du syndrome de Cush-

1. l'hyperinsulinisme

a) Les résultats déjà publiés chez l'homme $[33,15$, 16] font état d'un hyperinsulinisme. Ceci est en accord avec les données expérimentales utilisant la cortisone chez l'animal [5], le cortisol [26] ou la déxaméthasone [27] chez l'homme.

Histologiquement, l'administration de Cortisone permet d'observer une hypertrophie des îlots de Langerhans avec la disparition des granulations bêta [17].

b) Le mécanisme de cet hyperinsulinisme reste discuté; il peut être indirectement lié à l'hypercortisolisme par l'intermédiaire de l'hyperglycémie. L'hypercortisolisme, en effet, augmente le pool glucidique par néoglycogénèse accrue et par augmentation de production du glucose.

En faveur de cette hypothèse, on peut retenir l'expérience de Perley [27] qui montre qu'une discrète élévation glycémique, réalisée par perfusion continue d'une faible quantité de glucose est capable d'aug. menter la libération d'insuline sous tolbutamide, presqu'autant qu'un traitement court par la déxaméthasone, au cours duquel la glycémie basale était également discrètement augmentée. De plus, nos malades ont une augmentation de la réponse insulinique sous arginine, or, on sait que chez le sujet dont le pancréas est intact le glucose et l'arginine sont synergiques en ce qui concerne le relâchement d'insuline.

Il peut s'agir au contraire d'une action directe du cortisol sur la libération ou la sécrétion d'insuline:

chez l'animal en effet, [5] l'élévation insulinémique précède l'élévation glycémique lors de l'administration de cortisone.

Par ailleurs la sécrétion d'insuline in vitro induite par le glucose augmente significativement chez des animaux à qui l'on a administré du cortisol in vivo, alors même que il n'y a pas de changement dans le contenu global en insuline du pancréas de ces animaux; le cortisol augmenterait done la sensibilité de l'îlot pancréatique au glucose.

Chez nos malades il n'y a pas de parallélisme entre l'hyperinsulinisme et l'élévation de la glycémie à jeun. Le seule malade ayant une hyperglycémie provoquée normale (VII) a un hyperinsulinisme net. Enfin, le pic insulinémique peut être plus précoce que le pic glycémique. 
Les résultats de la pertusion d'arginine montrent $q u$ 'un stimulus apparemment direct et différent du glucose entraîne une augmentation insulinémique.

Enfin, une question soulerée par Karam [15] est celle du rôle joué par l'obésité dans la genèse de cet hyperinsulinisme. En effet, cet auteur ne retrouve d'hyperinsulinisme que dans les cinq cas où existait un excès de poids dépassant $20 \%$ du poids idéal. Nos résultats ne confirment pas ces données, et ce fait est du reste en désaccord avec les données expérimentales: perte de poids chez les animaux traités par cortisol $[5,21]$.

Seule une de nos malades est franchement obèse (XIII $=$ excès de poids de $20 \%$ d'après les statistiques de la Life Assurance Company) = Sa réponse insulinémique est très faible. La patiente II a un excès de poids de $12 \%$, elle n'a pas d'hyperinsulinisme. Parmi les cinq patients hyperinsulinémiques, une seule (I) a un discret excès de poids $(6 \%)$. Or son hyperinsulinisme disparaît après surrénalectomie alors que son poids est inchangé.

c) L'inefficacité de cet hyperinsulinisme endogène, (puisque le rapport insuline/glucose est souvent inférieur à celui des témoins), ainsi que l'augmentation habituelle de la tolérance de ces malades à l'insuline exogène reste inexpliquée.

L'inhibition de l'utilisation périphérique du glucose sous l'influence des glucocorticoïdes est évoquée par certains $[18,24,25,30]$ et niée par d'autres auteurs [7]. De plus, la dégradation de l'insuline n'est pas modifiée [8].

Ces faits expérimentaux suggèrent la prédominance de l'augmentation de la production de glucose dans la genèse du diabète stéroidien.

Cependant, l'existence de facteurs antagonistes de l'insuline ne peut pas être écartée. Parmi ceux-ci:

l'hormone de croissance ne peut être retenue comme le montrent les résultats de nos dosages radio-immunologiques de STH chez ces mêmes malades [35].

Les NEFA ont été trouvés normaux à jeun dans le Cushing [29] ou parfois légérement élevés [9]. La charge glucosée entraîne une chute un peu retardée des NEFA [9].

\section{L'hyperinsulinisme peut être absent.}

En effet, cinq de nos malades seulement sur treize ont un hyperinsulinisme. Les huit autres ont un rapport insuline/glucose abaissé. La réponse insulinique pour un même stimulus glycémique est donc plus faible que normalement, ce qui suggère un trouble sécrétoire.

Cette insuffisance sécrétoire se différencie de celle observée au cours des diabètes non obèses de la maturité par un pic insulinémique plus précoce que le pic glycémique chez certains de nos malades et une réponse à l'arginine normale sinon augmentée, à l'exception de la malade XIII, qui se comporte comme un diabète insulino prive. Il faut rappeler que la sensibilité à ce stimulus est précocément diminuée dans le diabète commun de l'adulte [10).
3. Nos résultats portent sur une population limitée de malades atteints d'un syndrome de Cushing. Ils montrent l'existence de troubles hétérogènes de l'insulino-sécrétion:

dans certains cas, on retrouve un hyperinsulinisme indépendamment de toute obésité,

ailleurs, existe un déficit insulinique par rapport au stimulus glycémique alors que la réponse à l'arginine est normale,

enfin, dans un de nos cas existait un diabète insulinoprive.

La discordance entre l'hétérogénéité des troubles de l'insulinosécrétion observée chez nos patients et les données expérimentales suggère l'existence d'autres facteurs pathogènes que le seul hypercortisolisme. L'absence de corrélation entre la durée apparente de la maladie et le type de réponse insulinémique ne supporte pas dans notre série l'hypothèse de l'évolution d'un stade initial d'hyperinsulinisme vers un stade de déficit sécrétoire.

\section{Conclusion}

Au cours de la charge glucosée par voie orale, les résultats des dosages d'insulinémie révèlent un trouble hétérogène de l'insulinosécrétion au cours du syndrome de Cushing.

Dans certains cas, on retrouve un hyperinsulinisme, conforme aux données expérimentales. Cet hyperinsulinisme est indépendant de l'obésité et semble relever plus de l'augmentation de production de glucose que de l'existence de facteurs antagonistes de l'insuline notamment hormonaux.

Dans d'autres cas, l'insulinémie n'apparaît pas différente de celle des sujets témoins, alors que les courbes glycémiques sont diabétiques, ce qui suggère une insuffisance sécrétoire. Ces sujets se différencient des diabétiques non obèses de la maturité par la réponse normale lors de la stimulation par l'arginine. Le mécanisme de cette insuffisance sécrétoire au cours du syndrome de Cushing est à l'étude.

Remerciements. Nous remercions Melle. A.M. Bridoux et Monsieur L. Pique pour leur collaboration technique, Melle J. Truffert pour les dosages des glycémies et Melle J. Vitiello pour la mise au point du texte.

Nous remercions également Monsieur le Professeur Gilbert Dreyfus qui nous a permis d'étudier une de ses malades.

\section{Bibliographie}

1. Ashmore, J.: Effects of glucocorticoids on insulin action. Diabetes 13, $249-353$ (1964).

2. Bassett, J.M., Wallace, A.C.C.: Influence of cortisol on plasma insulin on the sheep. Diabetes 16, 566-671 (1967).

3. Bastenie, A.: Cortico-surrénale et diabète humain. Vol. 1, p. 356. Paris: Masson 1956.

4. Bricaire, H., Leprat, J., Luton, J.P.: La thérapeutique des syndromes de Cushing. 9ème Congrès d'endocrinologio de langue Française. Montreal Septembre 1967 Paris: Masson. 
5. Campbell, J., Rastogi, K.S., Hansler, H.R.: Hyperinsulinemia with diabetes induced by cortisone and the influence of growth hormone in the Chinese Hamster. Endocrinology 79, $749-756$ (1966).

6. Conn, J.W., Fajans, S. S.: The prediabetic state. Amer. J. Med. 31, 839-849 (1961).

7. Debodo, R.C., Altszuler, N.: Insulin hypersensitivity and physiological insulin antagonists. Physiol. Rev. $38,389-445$ (1958).

8. Elgee, N.J., Williams, R.H.: Pituitary and adrenal influences on insulin $I^{131}$ degradation. Amer. $J$. Physiol. 180, 9-12 (1955).

9. Felt, V. Serum F.F.A. and other lipids during glucose loading in endocrinopathies. Amer. J. med. Sci. 254, $161-172$ (1967).

10. Floyd, J.C., Fajans, S.S., Conn, J.W., Thipparet, C., Knopf, R.F., Guntsche, E.: Secretion of insulin induced by amino acids and glucose in diabetes mellitus. J. clin. Endocr. 28, 266-276 (1968).

11. - - - Knopf, R.E., Rull, J.: Stimulation of insulin secretion by amino acids. J. clin. Invest. 45, 1487-1501 (1966).

12. Haist, R.E.: Factors affecting the insulin content of the pancreas. Physiol. Rev. 25, 409-444 (1944).

13. Hennemann, D.H., Bunker, I.P.: The pattern of intermediary carbohydrate metabolism in Cushing's syndrome. Amer. J. Med. 23, 34-45 (1957).

14. Ingle, D.J.: Experimental diabetes. Diabetes 9, 297 $-308(1960)$.

15. Karam, J.H., Grodsky, G.M., Forsham, P.H.: Relation-ship of obesity and G.H. to serum insulin levels. Ann. N. Y. Acad. Sci. 131, 374-380 (1965).

16. Klink, D., Estrich, D.: Plasma insulin concentration in Cushing's syndrome and thyrotoxicosis. Clin. Res. Proc. 12, 354 (1964).

17. Lazarus, S.S., Volk, B.W.: The pancreas in human and experimental diabetes. Vol. 1. New York: Grune and Stratton 1962.

18. Leboeuf, B., Renold, A.E., Cahill, G.F.: Studies on rat adipose tissue in vitro IX - Further effects of cortisol on glucose metabolism. J. biol. Chem. 237, 988-991. (1962).

19. Lebon, J., Lentenegger, M., Illoul, G.: Troubles de la glycorégulation et corticothérapie. (A propos de 22 observations.) Le Diabète 13, 139-145 (1965).

20. Levine, R.: Analysis of the actions of the hormonal antagonists of insulin. Diabetes 13, 362-365 (1964).

21. Malaisse, W.J., Malaisse-Lagae, F., Mac Graw, E.F., Wright, P.H.: Insulin secretion in vitro by pancreatic tissue from normal, adrenalectomized and cortisol treated rats. Proc. Soc. exp. Biol. 124, 924-928 (1967).

22. Miller, M.: Diabetes associated with other conditions Diabetes 11, 710-722 (1962).

23. Modigliani, E., Strauch, G., Delzant, G., Dreyfus, G.: Etude de la sécrétion d'insuline au cours de la perfusion d'arginine: effets comparés de sympatholytiques chez des sujets normaux et hyperthyroidiens Rev. franç. Et. clin. biol. 14, 267-275 (1969).

24. Morgan, H. E., Regen, D. M., Henderson, M. J., Sawyer, T.K., Parke, C.R.: Regulation of glucose uptake in muscle VI. Effects of hypophysectomy, adrenalectomy, growth hormone, hydrocortisone and insulin on glucose transport and phosphorylation in the perfused rat heart. J. biol. Chem. 236, 2162-2168 (1961).

25. Munk, A.: The effect of cortisol on glucose uptake by rat epididymal fat pads. Endocrinology 68, 178-180 (1961).

26. Perkoff, G.T., Parker, V., Mac Call, J.F., Tyler, F.H. : Early effects of cortisol on glucose metabolism in man. J. Lab. clin. Med. 62, $431-436$ (1963).

27. Perley, M.M., Kipnis, D.H.: Effects of glucocorticoids on plasma insulin. New Engl. J. Med. 274, 1234-1237 (1966).

28. Plotz, C.M., Knivlton, A.I., Ragan, L.: The natural history of Cushing's syndrome. Amer. J. Med. 13, $597-614$ (1952).

29. Pupo, A.A.: Carbohydrate metabolism in hyperadrenocorticism. Diabetes 15, 24-29 (1966).

30. Riddick, F.A., Reisler, D.M., Kipnis, D.M.: The sugar transport system in striated muscle; effect of growth hormone, hydrocortisone and alloxan diabetes. Diabetes 11, 171-178 (1962).

31. Romanoff, N.E.: Factors controlling release of insulin from beta cell in relation to diabetes mellitus. N.Y. med. J. 68, 385-391 (1968).

32. Rosselin, G., Dolais, J.: Application de la méthode radio-immunologique au dosage de l'insuline humaine et au dosage d'hormone folliculo-stimulante humaine. Monographie annuelle de la Société Française de Biologie Clinique, $189-217$ (1968).

33. Schwarz, P.: Verk. dtsch. Ges. inn. Med. 68, 289 (1962).

34. Soffer, J.L., Eisenberg, J., Ianaccone, A., Gabrilove, J.E.: Cushing's syndrome in the human adrenal cortex. Colloquia Ciba on Endocrinology 8, 487.

35. Strauch, G., Modigliani, E., Luton, J.P., Bricaire, H. : Partial somatotropin insufficiency in Cushing's syndrome. Acta endocr. 60, $121-129$ (1969).

36. Thorn, G.W., Renold, A.E., Cahill, G.F.: The adrenal and diabetes. Diabetes $8,337-343(1959)$.

37. - - Winegrad, A.I.: Some effects of adrenal cortical steroids on intermediary metabolism. Brit. med. J. 1957 II, 1009.

Dr. E. Modigliani

Laboratoire de Pathologie Médicale

de la Faculté de Médecine et

Service d'Endocrinologie et de

Métabolisme

Hôpital Cochin

F-75 Paris XIVe 